Article

\title{
Proton Acceleration by Ultrashort Intense Laser Interaction with Microstructured Snow Targets
}

Elad Schleifer ${ }^{1, *}$, Zohar Henis ${ }^{1}$, Mordechai Botton ${ }^{1}$, Omer Shavit ${ }^{1}$, Daniel F. Gordon ${ }^{2}$ and Arie Zigler ${ }^{1}$

1 Racah Institute of Physics, Hebrew University, Jerusalem 91904, Israel; E-Mails: zoharhenis@phys.huji.ac.il (Z.H.); bdmoti@mail.huji.ac.il (M.B.); omer.shavit@mail.huji.ac.il (O.S.); zigler@vms.huji.ac.il (A.Z.)

2 Plasma Physics Division, Naval Research Laboratory, Washington, DC 20375, USA; E-Mail: daniel.gordon@nrl.navy.mil

* Author to whom correspondence should be addressed; E-Mail: Elad.schleifer@mail.huji.ac.il; Tel.: +972-54-4646730; Fax: +972-2-6512483.

Academic Editor: Ken Ledingham

Received: 9 April 2015 / Accepted: 18 August 2015 / Published: 26 August 2015

\begin{abstract}
Enhanced proton acceleration to high energy by relatively modest ultrashort laser pulses and structured dynamic plasma snow targets was demonstrated experimentally. High proton yield emitted to narrow solid angle with energies of up $25 \mathrm{MeV}$ were detected from interaction of a $5 \mathrm{TW}$ laser with snow targets. The high yield was attributed to a carefully planned prepulse and microstructured snow targets. We studied experimentally the minimal energy requirements for the adequate prepulse and we are using PIC simulations to study the dynamics of acceleration process. Based on our simulations, we predict that using the proposed scheme protons can be accelerated to energies above $150 \mathrm{MeV}$ by $100 \mathrm{TW}$ laser systems.
\end{abstract}

Keywords: proton acceleration; laser plasma interaction; ultrashort intense laser; laser acceleration; laser driven radiation therapy

\section{Introduction}

Proton acceleration by the interaction of an ultra high intensity laser beam with matter is very important for basic science and has several wide prospective applications, including cancer treatment, 
nuclear physics and astrophysics in lab, (see [1-3] for review). In recent years, several promising acceleration schemes were suggested and demonstrated, among them Target Normal Sheath Acceleration (TNSA) [4,5], Radiation Pressure Acceleration (RPA) [6,7] collisionless shock acceleration [8] and Break Out Afterburner (BOA) [9]. A variety of schemes aimed to increase the interaction efficiency were proposed such as mass-limited targets [10] and nano-structure targets [11-13]. The above accelerating schemes are optimized for interaction between the high intensity main laser pulse and a cold solid-density target, and are strongly degraded as the main laser pulse interacts with a pre-heated or ionized target caused by a prepulse. Therefore, a huge experimental effort is focused in reducing the energy in the prepulse, i.e., contrast ratio of the order of $10^{-11}$ for $100 \mathrm{TW}$ laser system and even higher for more energetic systems.

Recently, we have demonstrated an alternative approach to laser-based proton acceleration by using a moderate power laser system and micro-structured snow targets [14-16]. These targets were engineered by depositing snow on sapphire substrates. It was shown that pre-formed plasma formed by a prepulse may be beneficial to the acceleration process $[17,18]$. Our experimental results [16] show that the energy of the accelerated protons scales with the power of the laser according to $E_{p} \sim P_{L}^{1 / 2}$, like other schemes [19] (see Figure 1). Using structured snow targets and relatively low contrast ratio this scaling was demonstrated for significantly lower laser powers than the traditional schemes [14,18]. Moreover, in experiments that were carried out at laser system with high contrast ratio (short pulse prepulse with energy below microjoule), where the main pulse interacted directly with solid snow surface, the protons were accelerated to much lower energy [14-16]. The high proton energy can be attributed to combination of several effects: The improved laser absorption [18] and the interaction of the laser with mass limited plasma with density gradient induced by the prepulse lead to significant enhancement of its effective electrical field [16]. In addition, field enhancement at the tip of snow micro-structures and their curved surface may enhance as well the interaction, leading to higher proton energies.

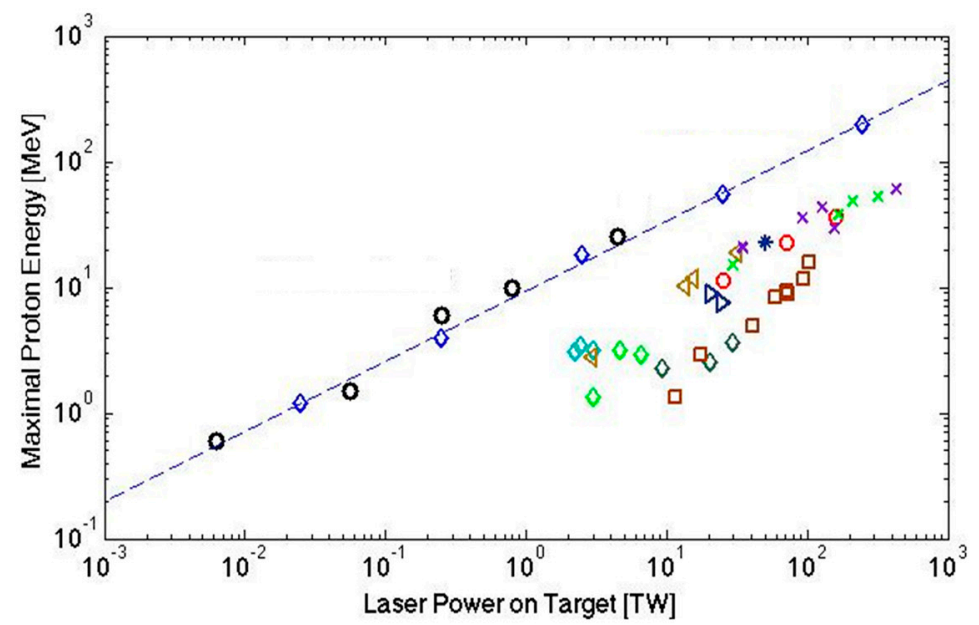

Figure 1. Energy scaling of the accelerated protons as a function of the laser power on target. The various points describe data obtained by many contributors (reproduced from [19]), the experimental results obtained with snow targets are marked by black circles. Results of PIC simulations are marked by blue diamonds (Reproduced with permission from [16]; published by American Physical Society, 2013). 
Numerical 2D particle in cell (PIC) code simulations [16], with initial conditions given by the state of the plasma snow at the time of the interaction with the main pulse in the experiments, reproduced the obtained proton energy and explained the crucial role of the dynamic plasma produced by prepulse illumination of the snow target. For laser powers greater than $100 \mathrm{TW}$, the simulation predicts proton energy near $150 \mathrm{MeV}$.

In this paper, we further explore the role of the dynamic plasma generated by the prepulse in our approach to laser-induced proton acceleration based on snow structured targets and moderate power $(<10 \mathrm{TW})$ laser systems. These aspects of the work are published for the first time. Section 2 describes the morphology of the snow targets and presents measurements of their optimal damage threshold (ODT) aimed to understanding and controlling the prepulse produced plasma parameters. Section 3 displays results of PIC simulations of the laser interaction with snow micro-structured targets, which support our proton acceleration approach. The paper is concluded in Section 4.

\section{Properties of Microstructured Snow Targets}

The snow structured targets were generated on liquid nitrogen cooled sapphire substrate on which water vapor was deposited. The snow structure is determined by parameters of growth conditions, such as pressure, flow velocity, and temperature, and by the nucleation centers [20,21].

The snow surface can be described as a highly structured surface with three roughness scales: (1) pillars in the scale of $100 \mu \mathrm{m}$; (2) spikes in the length of $10 \mu \mathrm{m}$ on top of them; and (3) whiskers in the scale of $1 \mu \mathrm{m}$ on top of the spikes. The distance between the structures is of the order of their roughness scales. A key factor determining the interaction of the main pulse with the snow target is how its highly structured surface is affected by prepulse or lower pedestals intensity levels irradiation, in particular, whether each roughness scale prevails and how steep of the plasma gradient generated by the prepulse is.

One of the parameters determining the state of the target after the interaction with the prepulse and the plasma density gradient at the interaction of the main pulse with the target is the Optical Damage Threshold (ODT). As it will be shown here, the ODT intensity level is lower than the prepulse intensity; however, it represents a lower limit for controlling the level of possible existing pedestal in the laser system. This feature is identified in the experiments reported here by irreversible change of the surface [22]. The ODT is a consequence of multiphoton followed by impact ionization of the snow [23-26]. The free electrons generated by ionization escape the target and due to charge separation they pull the ions out of the target.

In order to measure the prepulse influence on the microstructure targets with tens of femtoseconds gate resolution and a few microns for space resolution we used an imaging system and gating technique [22].

The measurements of the ODT of snow were performed at the Hebrew University High Intensity Laser Facility with a Ti: Sapphire laser based on Chirped Pulse Amplification (CPA) that can deliver peak power of $1 \mathrm{TW}$ in $50 \mathrm{fsec}$ with $10 \mathrm{~Hz}$ repetition rate.

In order to determine the snow ODT, the laser energy on target was controlled using filters and reduced to the prepulse intensity level. The diagnostic method was strobe illumination by short pulse white light source and imaged at three times: 
1. Before the interaction of the laser with the snow target.

2. $7 \mathrm{~ns}$ after the interaction (around the delay between the prepulse and the main pulse).

3. At longer times, minutes after the interaction.

The target was imaged at these three times with a telescope system to a CCD camera placed outside the vacuum chamber. The diagnostic beam was part of the main beam that was used for generating super-continuum strobe, enabling gives a controllable delayed probing with fast gating at the time scale of the laser pulse duration. The measurements were conducted for various values of the laser fluence on target, in the range $(0.1-3) \mathrm{J} / \mathrm{cm}^{2}$.

Three images were recorded for each laser fluence (Figure 2). The first image of the snow target, long before the interaction, was obtained by blocking the main beam and using the strobe alone. The second image, 7 nanoseconds after the interaction, of the order of the time duration between the prepulse and the main pulse of the laser system, was obtained using both beams of the laser, and the third image, long after the interaction, again by blocking the main beam and using the probe beam only.

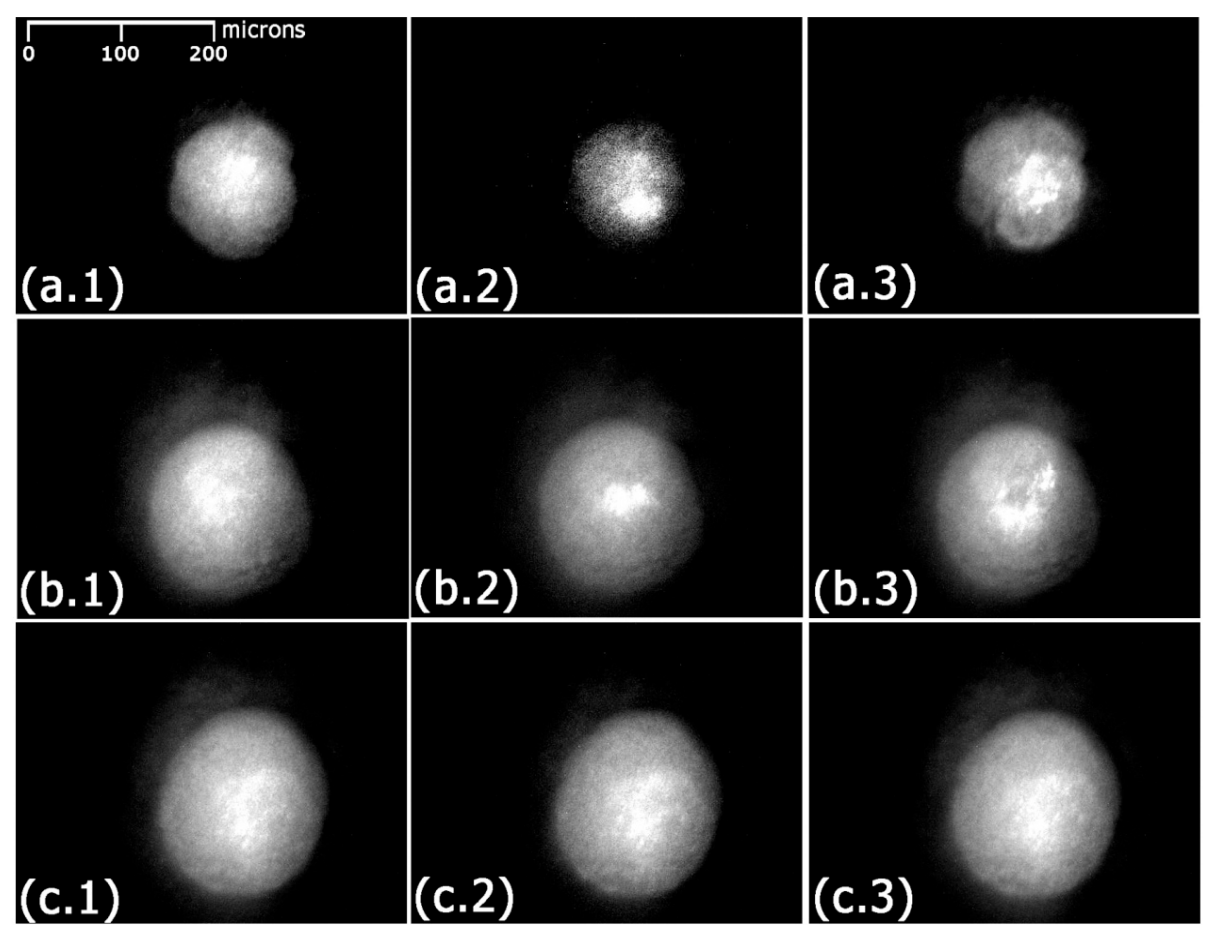

Figure 2. Strobe images of the snow microstructures at different laser fluences and at different times according to laser-snow interaction. (a.1-3) laser at $1 \mathrm{~J} / \mathrm{cm}^{2} ;(\mathbf{b . 1}-\mathbf{3})$ at $0.45 \mathrm{~J} / \mathrm{cm}^{2}$ and (c.1-3) at $0.35 \mathrm{~J} / \mathrm{cm}^{2}$; (a-c.1) strobe image before the interaction; (a-c.2) an image $7 \mathrm{~ns}$ after the interaction and (a-c.3) long after the interaction, few minutes).

Damage was identified as irreversible change in the target surface and was identified by measuring the intensity as a function of distance along lines on the image. Using this processing procedure, we have seen that at fluence higher than $1 \mathrm{~J} / \mathrm{cm}^{2}$ on target a definite damage has been occurred to the target, as can be seen in Figure 2 (a.1-3). At laser fluence on target less than $0.35 \mathrm{~J} / \mathrm{cm}^{2}$ we could not see any damage with our imaging system and processing procedure (Figure 2 (c.1-3)). At fluence of 
the laser on the target of $0.45 \mathrm{~J} / \mathrm{cm}^{2}$ we can hardly see any damage 7 nanosecond after the interaction (Figure 2 (b.2)); though long after the interaction due to thermal conductivity it is obvious that ablation occurred (Figure 2(b.3)). From this result, we concluded that the laser fluence threshold for optical damage ODT is around $0.4 \mathrm{~J} / \mathrm{cm}^{2}$. this is in good agreement with a calculation the value calculated following Gamaly et al. [25].

Based on the above measured value of the damage threshold, the laser system that will be used for the future experiments with the micro-structured targets must suppress unwanted prepulse to below this level.

\section{Particle in Cell Simulations of High Intensity Laser Pulses with Snow Whiskers}

Numerical 2D PIC code simulations of the main laser pulse with the pre-formed plasma generated by the prepulse explain the role of the plasma density gradient and were found in agreement with the experimental scaling law shown in Figure 1. The increased proton energy may result from localized enhancement of the laser field intensity near the tip of the microstructured whisker. In addition, in the mass-limited whisker target mass-limited like phenomenon, i.e., the absence of a high density cold electron cloud in the vicinity of the whisker cannot compensate for the expelled electrons. The heated electrons remain in the vicinity of the positively charged whisker, and strong accelerating electrostatic fields are produced, pulling the protons out. The Coulomb explosion of the positively charged whisker, add longtime acceleration to the protons. A one dimensional fluid model, demonstrating the field enhancement by an order of magnitude by the local plasma density near the whisker tip, was presented in our previous work [15].

Aimed to optimize the laser-snow interaction and enhance proton acceleration, we conducted a multi-variables numerical study of laser-snow structured target interaction, using the 3D particle in cell (PIC) code TURBOWAVE [27,28]. We considered intense laser pulse interaction with snow whiskers with different sizes, aspect ratios from 1 to 100, planar and ellipsoid shapes, density from step-like solid density to under-dense plasma with smooth Gaussian density gradients. Electrons, protons and oxygen ions acceleration was examined for laser intensity in the range $2.5 \cdot \times 10^{17} \mathrm{~W} / \mathrm{cm}^{2}$ to $2.5 \cdot \times 10^{21} \mathrm{~W} / \mathrm{cm}^{2}$ for different angles between the laser propagation and the whisker axis of symmetry. Our simulations show that maximum proton acceleration may occur due to combination of optimal conditions of the whisker's density gradient, surface curvature, aspect ratio and illumination conditions.

The laser spot size in our experiments was about 4-5 $\mu \mathrm{m}$, therefore the laser spot may have interacted with one or sequentially with few whiskers, based on the SEM characterization of the snow targets [20,21]. Here, for simplicity, we consider interaction with a single snow whisker. Following the prepulse illumination, the whisker is partially vaporized and ionized hence a non-uniform plasma cloud is formed. To study the influence of plasma density gradients on proton acceleration we model the non-uniform plasma cloud composed of protons, triply ionized oxygen ions and electrons, as an ellipsoid. We assumed that the snow whisker consists of a solid core surrounded by gradually decreasing density plasma, such that the critical density region is located at a distance larger by about a factor of 10 than the width of the solid core. An ellipsoid shape density enables as well study of expected laser-snow interaction enhancement with curved surfaces, in particular near the tip of the whisker [15], extending previous studies that considered only spherical and cylindrical targets [29]. 
Figure 3 shows the electron density distribution and the laser beam before the interaction for one of the cases considered in our simulations. In this example, the high density part of the whisker, of $100 \cdot n_{\mathrm{cr}}$, where $n_{\mathrm{cr}}=1.71 \times 10^{21} \mathrm{~cm}^{-3}$ is the critical density at laser wavelength $0.8 \mu \mathrm{m}$, is an ellipsoid of the order of $0.1-0.2 \mu \mathrm{m}$ width (minor axis) and 1-2 $\mu \mathrm{m}$ length (major axis), and the critical density contour is an ellipsoid of the order of 1-2 $\mu \mathrm{m}$ width (minor axis) and $10 \mu \mathrm{m}$ length (major axis). Our simulations assume that the polarization vector of the laser electric field and the major axis of the whisker are in the same plane.

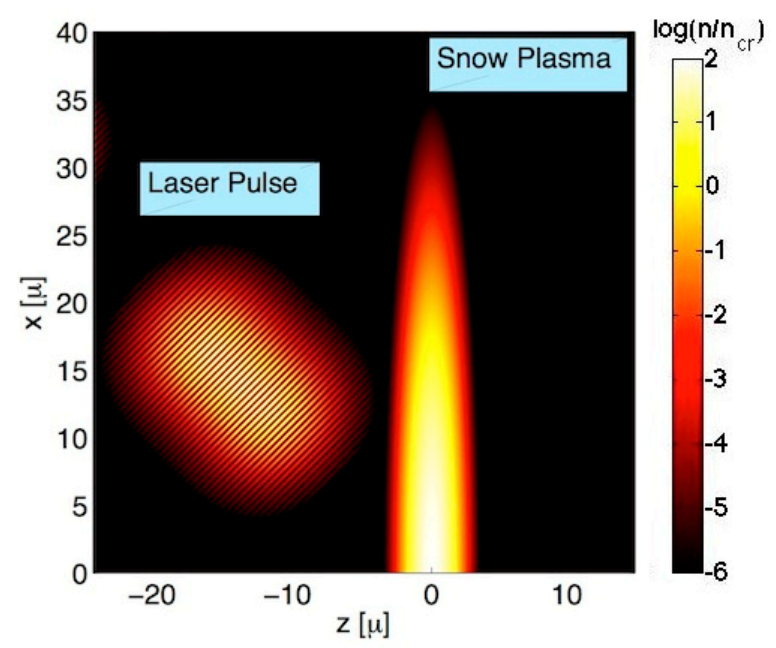

Figure 3. Spatial distribution of the electron plasma of the whisker before the start of the interaction with the main pulse. The upper half of the whisker is displayed. The density of the plasma is logarithmic scaled by the critical density. One can see a central dense core, surrounded by gradually lower density region. The laser electric field is schematically shown and not to scale (Adapted with permission from [16], published by American Physical Society, 2013).

To explore the influence of the plasma density, density gradients and surface curvature we simulated the laser-whisker interaction for different values of whisker aspect ratios and density gradients. For comparison, we considered planar shape and constant solid density whiskers as well.

To obtain the scaling law of the protons energy with the laser intensity, our multi-parameters simulations considered different laser intensities in the range $2.5 \times 10^{17} \mathrm{~W} / \mathrm{cm}^{2}$ to $2.5 \times 10^{21} \mathrm{~W} / \mathrm{cm}^{2}$, corresponding to normalized vector potential $a_{0}=\frac{e \cdot E}{m \cdot c \cdot \omega}$ in the range 0.34 to 34 . A laser pulse of $88 \mathrm{fs}$ total duration with linear rise time and fall time of $32 \mathrm{fs}$ each, wavelength $\lambda$ of $0.8 \mu \mathrm{m}$ and spot size of $4 \mu \mathrm{m}$ was used in the simulations.

An example of the particles distribution at different times after the laser-whisker interaction is shown in Figure 4. The laser pulse at intensity $2.5 \times 10^{19} \mathrm{~W} / \mathrm{cm}^{2}\left(\mathrm{a}_{0}=3.4\right)$ propagates perpendicular to the major axis of an ellipsoid whisker of the type shown in Figure 5a with density gradient described before. The beam has Gaussian spatial distribution and its center is few microns above the middle of the major axis of the whisker (the center of the simulation box). The simulation region is $100 \mu \mathrm{m} \times 60 \mu \mathrm{m}$. Spatial density distributions of the electrons (left), protons (middle) and oxygen ions (right) are shown in Figure 5 at three different times, $212 \mathrm{fs}, 424 \mathrm{fs}$ and $850 \mathrm{fs}$. The laser pulse enters the simulation box from the left and hits the whisker at time $t=141 \mathrm{fs}$. As seen in the upper row of Figure 5, the electrons 
are accelerated by the laser's pondermotive potential. Part of the electrons escape out of the initial plasma cloud, generating a spherical low density cloud with accumulation near the tip of the whisker, which induces charge separation and generates an electrostatic field. We notice that the density distribution is not fully symmetrical relatively to the minor axis of the whisker, as the laser hits the whisker above its center of symmetry and is reflected from curved surfaces. It is seen from the electrons density distribution that there is not clear distinction between the back and the front surfaces as there are mixed over the whisker's tip, unlike in the TNSA mechanism. The middle row of Figure 5 displays the particles density distribution at time $t=424 \mathrm{fs}$. It is seen that a significant part of the electrons have left the initial whisker cloud. The charge separation is higher and the protons are accelerated by the electrostatic field. The asymmetry relatively to the whisker's minor axis is observed in the protons density distribution as well. The charge separation between the hot electron cloud and the positively charged whisker generates also an accelerating field in the lateral direction, and the total amplitude of the field is enhanced. At this time the heavier oxygen ions are starting to react to the electrostatic field and their density distribution is disturbed. The bottom row of Figure 5 displays the density distribution at time $t=850 \mathrm{fs}$. The electrons and protons distributions have highly expanded. The density distribution of the electrons near the two tips looks similar, their accumulation is reduced and decreases gradually over all directions. A sharp front is observed in the density distribution of the protons and the oxygen ions. Their upper side density distribution is wider. Few "hole"-like spots are seen in the protons density distribution. Charge separation between electrons and ions is maintained, continuing the protons acceleration, while the un-neutralized heavier oxygen ions remain behind and add a pushing field. At further longer times, the charge separation diminishes, the acceleration stops and the protons move at constant velocity.

Figure 4 displays the structure of the electric field at early time, $235 \mathrm{fs}$ after the laser hit the whisker. At the bottom left corner the bright yellow region represents the electric field of the laser surrounded by a cloud of electrons. The field associated with the electrons is seen as an external weak region, due to the first part of electrons expelled out of the whisker and an inner spherical shell. Inside this shell, there is a double ellipsoidal shell associated with the protons front, outer shell and, inner shell with size similar to the whisker before the interaction, associated with the oxygen ions, that almost did not move by that time.

To emphasize the role of the special geometry and density gradient for the enhancement of laser-snow whisker interaction, Figure 6 shows four whisker shapes: Figure 6A is the whisker considered in the figures above; Figure 6B has ellipsoidal shape, to capture the effect of mass limited curved target, and constant solid density; Figure $6 \mathrm{C}$ is similar to $\mathrm{A}$, but longer, to mimic interaction of the laser with plasma gradient, but planar surface; and Figure 6D has solid density and planar shape, similar to targets where the protons acceleration is driven by the TNSA mechanism. 


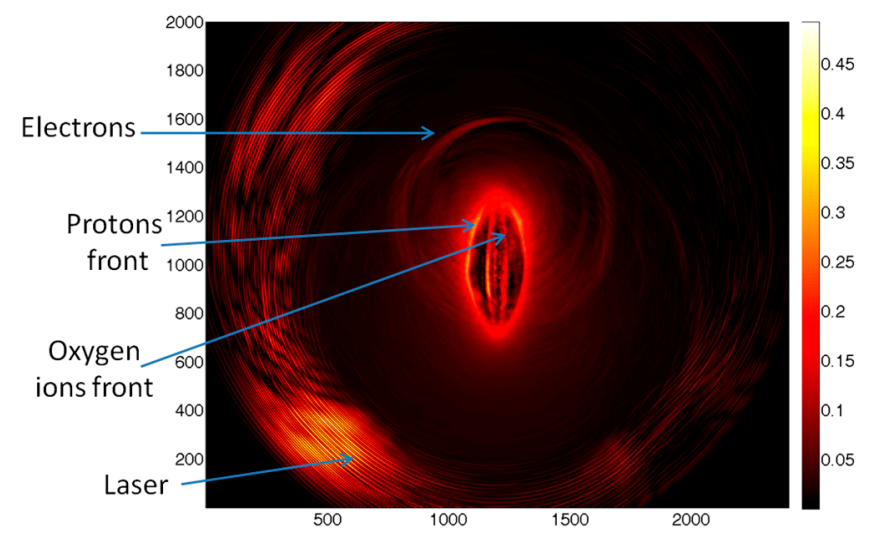

Figure 4. Spatial dependence of the electric field in units of a $0=3.4$, at $235 \mathrm{fs}$ after the start of interaction of a laser pulse at intensity $2.5 \times 10^{19} \mathrm{~W} / \mathrm{cm}^{2}$ at $45^{\circ}$ irradiation angle.
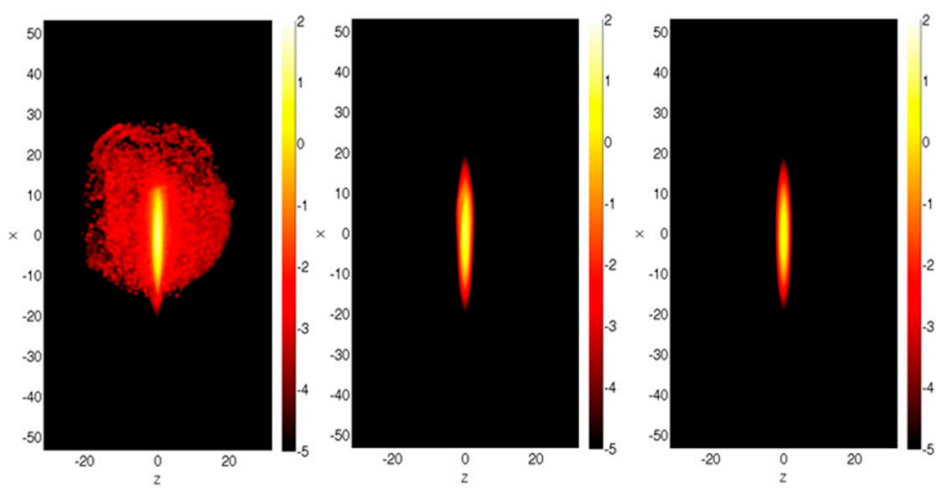

$a=212 \mathrm{fs}$
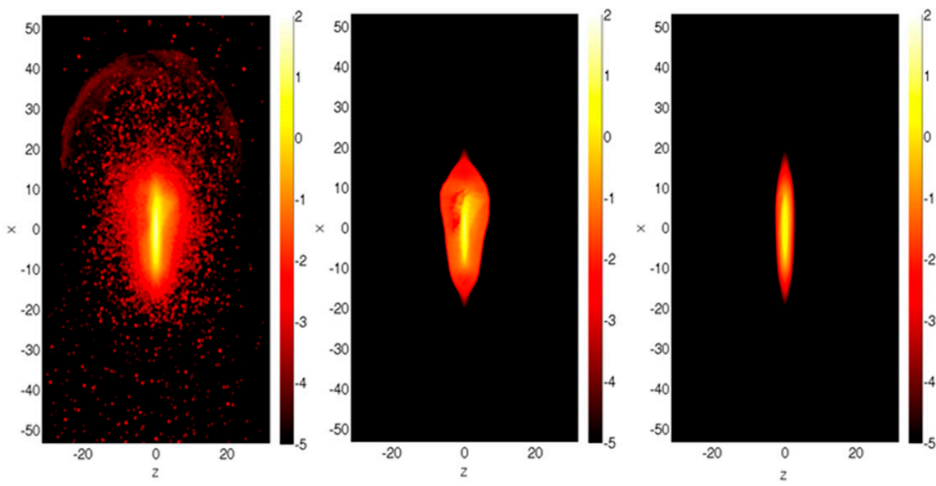

$$
b=424 \mathrm{fs}
$$
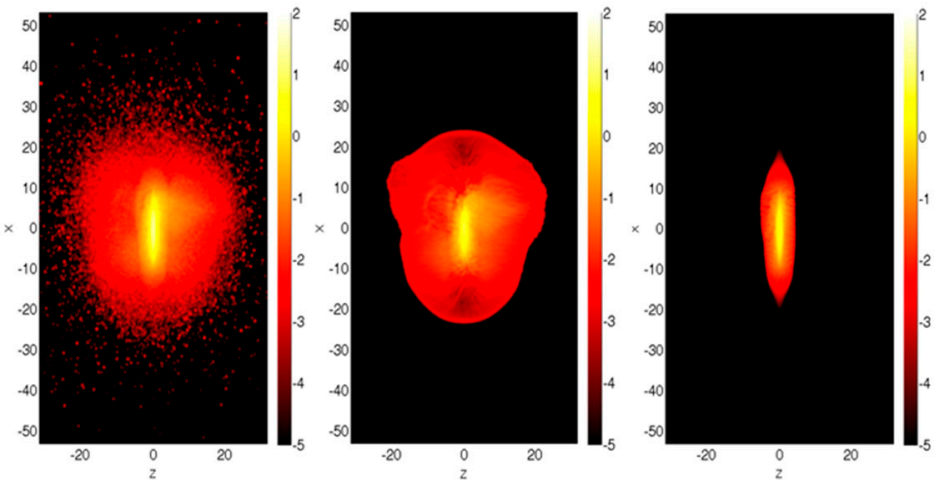

$\mathrm{c}=850 \mathrm{fs}$

Figure 5. Spatial density distribution of electrons (left); protons (middle); oxygen ions (right) at times a-212 fs, b-424 fs and c-850 fs. The laser hit the whisker at $141 \mathrm{fs}$. Color map scale for density is logarithmic normalized to critical density. Vertical ( $x$ ) and horizontal $(\mathrm{z})$ coordinates are given in microns. 


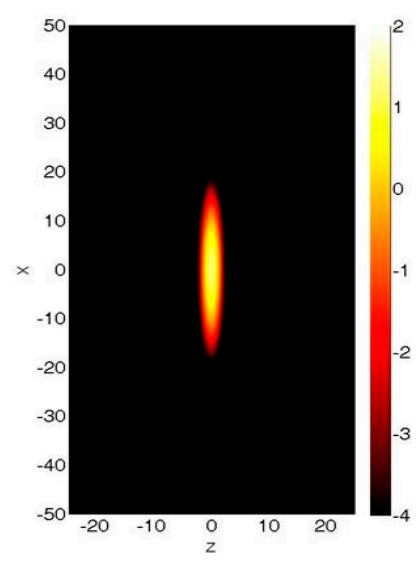

A

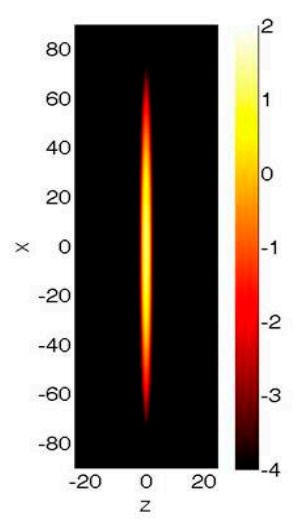

C

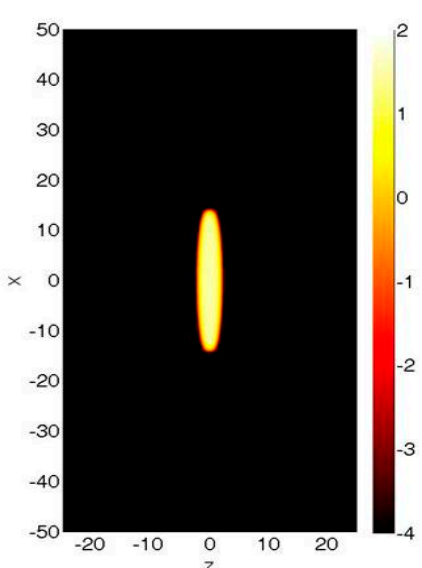

$\stackrel{z}{B}$

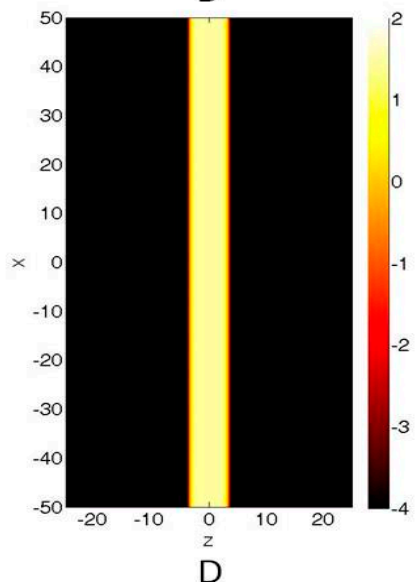

Figure 6. Various whisker models considered in the simulations, with the following properties: (A) ellipsoidal shape and density gradient; (B) ellipsoidal shape and constant density; (C) density gradient and approximately planar shape; (D) constant density and planar shape. X, Y axes are in $\mu \mathrm{m}$. Color map scale is logarithmic normalized related to the critical density.

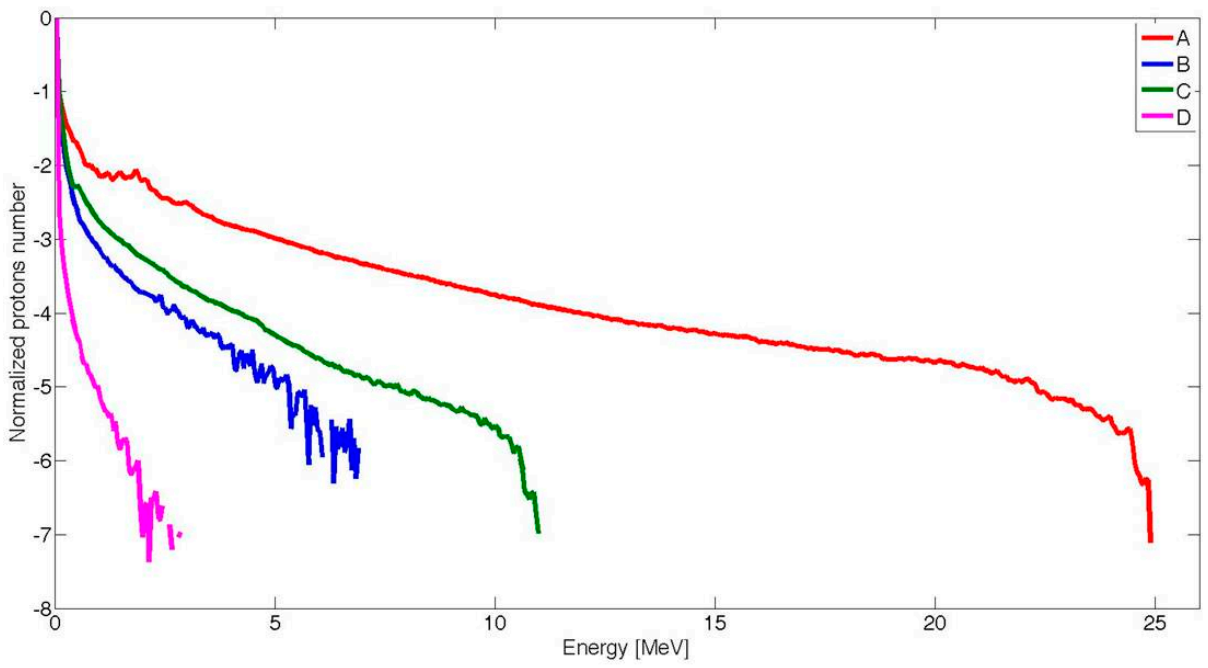

Figure 7. Simulated proton energy spectra for the cases defined in Figure 6.

The laser-snow whisker enhancement of the interaction, resulting from improved laser absorption, field enhancement near the tip and significantly larger charge separation for the case A, induces proton 
acceleration to much higher energies. The spectrum of the accelerated protons, calculated for the same laser intensity and irradiation angle, is shown in Figure 7, for the different whisker shapes shown in Figure 6.

The effect of the initial plasma whisker density gradient on the charge separation is further seen in Figure 8, comparing the electrons and protons distributions for cases A and B. At time about 320 fs after the start of the interaction, the electrons spatial distribution (Figure 8, left) is almost similar to the protons spatial distribution (Figure 8, right), resulting in much weaker charge separation.
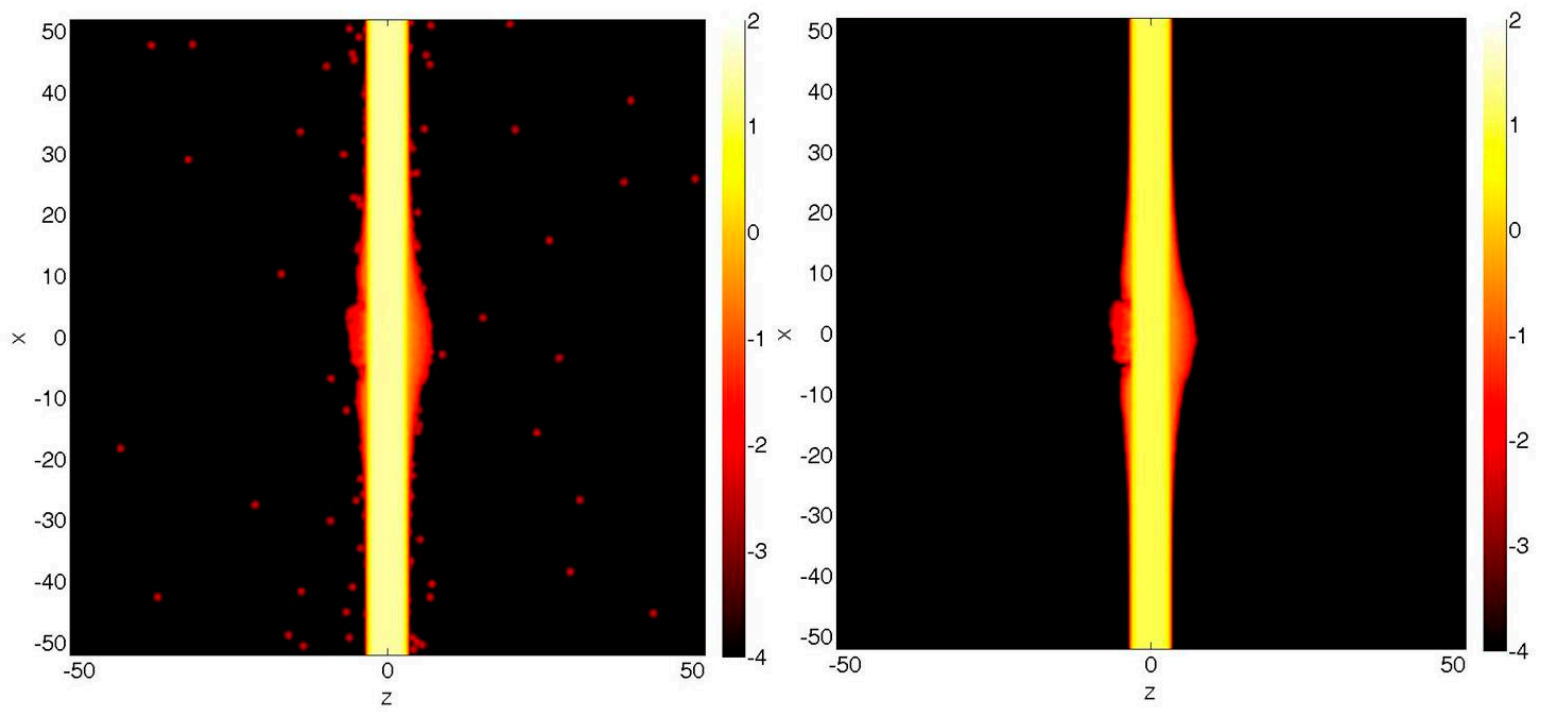

Figure 8. Electrons (left) and protons (right) spatial distributions obtained by simulation of the interaction of a laser pulse of intensity $2.5 \times 10^{19} \mathrm{~W} / \mathrm{cm}^{2}$, with ellipsoidal shape constant density whisker. $\mathrm{X}, \mathrm{Y}$ axes are in $\mu \mathrm{m}$. Color map scale is logarithmic normalized related to the critical density.

\section{Conclusions}

In conclusion, we have shown that our proton acceleration scheme might be a considerable improvement over alternative acceleration schemes, since it provides proton energies higher than anticipated based on TNSA with thin planar targets. Moreover, it requires lower energy laser system and relax the constraint of very high contrast ratio.

The targets were engineered by depositing snow on sapphire substrates, generating a microstructured morphology that can be controlled by two means: selective growth was achieved by deposition of artificial nucleation centers, while the lengths and shapes of the structures were controlled by varying the water vapor deposition rate. As in the acceleration mechanism considered here, the dynamic plasma generated by the pre-pulse is beneficial, aimed to understanding the properties of this plasma, we measured the ODT of the microstructured snow surface.

A multi-variables PIC simulations study was conducted to understand the above acceleration mechanism. Our findings are that the notably increased proton energy may be was attributed to a combination of several mechanisms, such as localized enhancement of the laser field intensity near the tip of the whisker, mass-limited like phenomenon and Coulomb explosion of the positively charged whisker. All these mechanisms are function of the shape, dimensions and local density profile of the 
snow whisker, the geometry of the irradiation and the laser intensity. Our simulations predict that $150 \mathrm{MeV}$ protons needed for medical applications can be produced using a $100 \mathrm{TW}$ laser system.

Further research will focus on experiments with more controlled and also periodic microstructured targets and explore the properties of their dynamic plasma generated by the prepulse. In addition, we are aimed to investigate whether this proton acceleration scheme could be applicable in proton therapy and other scientific uses.

\section{Acknowledgments}

The authors would like to acknowledge S. Eisenmann, E. Nahum, Y. Katzir, A. Baspaly, I. Pomerantz, F. Abicht, J. Branzel, G. Priebe, S. Steinke, A. Andreev, M. Schnuerer, W. Sandner, S.A. Pikuz and T. Palchan for their contribution to the results presented in Figure 1.

\section{Author Contributions}

The experimental data were collected by E. Schleifer, O. Shavit and A. Zigler. The theoretical analysis and PIC simulations were conducted by M. Botton, Z. Henis and D. Gordon.

\section{Conflicts of Interest}

The authors declare no conflict of interest.

\section{References}

1. Ledingham, K.W.D.; Bolton, P.R.; Shikazono, N.; Ma, C.M.C. Towards laser driven hadron cancer radiotherapy: A review of progress. Appl. Sci. 2014, 4, 402-443.

2. Fernández J.C.; Albright, B.J.; Beg, F.N.; Foord, M.E.; Hegelich, B.M.; Honrubia, J.J.; Roth, M.; Stephens, R.B.; Yin, L. Fast ignition with laser-driven proton and ion beams. Nucl. Fusion 2014, 54, 054006:1-054006:36.

3. Macchi, A. Ion acceleration by superintense laser plasma interaction. Rev. Mod. Phys. 2013, 85, 71.

4. Hatchett, S.P.; Brown, C.G.; Cowan, T.E.; Henry, E.A.; Johnson, J.S.; Key, M.H.; Koch, J.A.; Langdon, A.B.; Lasinski, B.F.; Lee, R.W.; et al. Electron, photon, and ion beams from the relativistic interaction of petawatt laser pulses with solid targets. Phys. Plasmas 2000, 7, 2076-2082.

5. Wilks, S.C.; Langdon, A.B.; Cowan, T.E.; Roth, M.; Singh, M.; Hatchett, S.; Key, M.H.; Pennington, D.; MacKinnon, A.; Snavely, R.A. Energetic proton generation in ultra-intense laser-solid interactions. Phys. Plasmas 2001, 8, 542-549.

6. Esirkepov, T.; Borghesi, M.; Bulanov, S.V.; Mourou, G.; Tajima, T. Highly efficient relativistic-ion generation in the laser-piston regime. Phys. Rev. Lett. 2004, 92, doi:10.1103/PhysRevLett.92.175003.

7. Hegelich, B.M.; Albright, B.J.; Cobble, J.; Flippo, K.; Letzring, S.; Paffett, M.; Ruhl, H.; Schreiber, J.; Schulze, R.K.; Fernandez, J.C. Laser acceleration of quasi-monoenergetic MeV ion beams. Nature 2006, 439, 441-444.

8. Haberberger, D.; Tochitsky, S.; Fiuza, F.; Gong, C.; Fonseca, R.A.; Silva, L.O.; Mori, W.B.; Joshi, C. Collisionless shocks in laser-produced plasma generate monoenergetic high-energy proton beams. Nat. Phys. 2012, 8, 95-99. 
9. Henig, A.; Kiefer, D.; Markey, K.; Gautier, D.C.; Flippo, K.A.; Letzring, S.; Johnson, R.P.; Shimada, T.; Yin, L.; Albright, B.J.; et al. Enhanced laser-driven ion acceleration in the relativistic transparency regime. Phys. Rev. Lett. 2009, 103, 045002.

10. Steinke, S.; Henig, A.; Schnuerer, M.; Sokollik, T.; Nickles, P.V.; Jung, D.; Kiefer, D.; Hoerlein, R.; Schreiber, J.; Tajima, T.; et al. Efficient ion acceleration by collective laser-driven electron dynamics with ultra-thin foil targets. Laser Part. Beams 2010, 28, 215-221.

11. Schwoerer, H.; Pfotenhauer, S.; Jackel, O.; Amthor, K.U.; Liesfeld, B.; Ziegler, W.; Sauerbrey, R.; Ledingham, K.W.D.; Esirkepov, T. Laser-plasma acceleration of quasi-monoenergetic protons from microstructured targets. Nature 2006, 439, 445-448.

12. Margarone, D.; Klimo, O.; Kim, I.J.; Prokupek, J.; Limpouch, J.; Jeong, T.M.; Mocek, T.; Psikal, J.; Kim, H.T.; Proska, J.; et al. Laser-driven proton acceleration enhancement by nanostructured foils. Phys. Rev. Lett. 2012, 109, 234801.

13. Andreev, A.; Kumar, N.; Platonov, K.; Pukhov, A. Efficient generation of fast ions from surface modulated nanostructure targets irradiated by high intensity short-pulse lasers. Phys. Plasmas 2011, 18, 103103.

14. Palchan, T.; Henis, Z.; Faenov, A.Y.; Magunov, A.I.; Pikuz, S.A.; Gasilov, S.V.; Skobelev, I.Y.; Zigler, A. Generation of fast ions by an efficient coupling of high power laser into snow nanotubes. Appl. Phys. Lett. 2007, 91, 251501.

15. Zigler, A.; Palchan, T.; Bruner, N.; Schleifer, E.; Eisenmann, S.; Botton, M.; Henis, Z.; Pikuz, S.A.; Faenov, A.Y., Jr.; Gordon, D.; et al. 5.5-7.5 MeV proton generation by a moderate-intensity ultrashort-pulse laser interaction with $\mathrm{H}_{2} \mathrm{O}$ nanowire targets. Phys. Rev. Lett. 2011, 106, 134801.

16. Zigler, A.; Eisenman, S.; Botton, M.; Nahum, E.; Schleifer, E.; Baspaly, A.; Pomerantz, I.; Abicht, F.; Branzel, J.; Priebe, G.; et al. Enhanced proton acceleration by an ultrashort laser interaction with structured dynamic plasma targets. Phys. Rev. Lett. 2013, 110, 215004.

17. Holkundkar, A.R.; Gupta, N.K. Effect of initial plasma density on laser induced ion acceleration. Phys. Plasmas 2008, 15, 123104.

18. Palchan, T.; Pecker, S.; Henis, Z.; Eisenmann, S.; Zigler, A. Efficient coupling of high intensity short laser pulses into snow clusters. Appl. Phys. Lett. 2007, 90, doi:10.1063/1.2435348.

19. Zeil, K.; Kraft, S.D.; Bock, S.; Bussmann, M.; Cowan, T.E.; Kluge, T.; Metzkes, J.; Richter, T.; Sauerbrey, R.; Schramm, U. The scaling of proton energies in ultrashort pulse laser plasma acceleration. New J. Phys. 2010, 12, 045015:1-045015:16.

20. Schleifer, E.; Nahum, E.; Botton, M.; Henis, Z.; Zigler, A. Control of snow morphology for laser-based accelerator targets. J. Phys. D-Appl. Phys. 2015, 48, doi:10.1088/0022-3727/48/8/085502.

21. Schleifer, E.; Botton, M.; Nahum, E.; Eisenman, S.; Zigler, A.; Henis, Z. Density measurements of laser interaction with ordered structured 'snow' targets. High Power Laser Sci. Eng. 2014, 2, e15.

22. Shavit, O.; Ferber, Y.; Papeer, J.; Schleifer, E.; Botton, M.; Zigler, A.; Henis, Z. Femtosecond laser-induced damage threshold in snow micro-structured targets. Appl. Phys. B: Lasers Opt., in press.

23. Stuart, B.C.; Feit, M.D.; Herman, S.; Rubenchik, A.M.; Shore, B.W.; Perry, M.D. Optical ablation by high-power short-pulse lasers. J. Opt. Soc. Am. B 1996, 13, 459-468.

24. Louzon, E.; Henis, Z.; Pecker, S.; Ehrlich, Y.; Fisher, D.; Fraenkel, M.; Zigler, A. Reduction of damage threshold in dielectric materials induced by negatively chirped laser pulses. Appl. Phys. Lett. 2005, 87, 241903. 
25. Gamaly, E.G.; Rode, A.V.; Tikhonchuk, V.T.; Luther-Davies, B. Electrostatic mechanism of ablation by femtosecond lasers. Appl. Surf. Sci. 2002, 197, 699-704.

26. Perry, M.D.; Stuart, B.C.; Banks, P.S.; Feit, M.D.; Yanovsky, V.; Rubenchik, A.M. Ultrashort-pulse laser machining of dielectric materials. J. Appl. Phys. 1999, 85, 6803-6810.

27. Gordon, D.F. Improved ponderomotive guiding center algorithm. IEEE Trans. Plasma Sci. 2007, $35,1486-1488$.

28. Gordon, D.F.; Mori, W.B.; Antonsen, T.M. A ponderomotive guiding center particle-in-cell code for efficient modeling of laser-plasma interactions. IEEE Trans. Plasma Sci. 2000, 28, 1224-1232.

29. Limpouch, J.; Psikala, J.; Andreev, A.A.; Platonov, K.YU.; Kawata, S. Enhanced laser ion acceleration from mass limited targets. Laser Part. Beams 2008, 26, 225-234.

(C) 2015 by the authors; licensee MDPI, Basel, Switzerland. This article is an open access article distributed under the terms and conditions of the Creative Commons Attribution license (http://creativecommons.org/licenses/by/4.0/). 